\title{
Importancia del peso de la oveja al parto en el comportamiento predestete de corderos Pelibuey
}

\author{
Importance of ewe body weight at lambing on pre-weaning performance \\ of Pelibuey lambs \\ José Alfonso Hinojosa-Cuéllar ${ }^{1,5}$, Jorge Oliva-Hernández ${ }^{1,2}$, \\ José Candelario Segura-Correa ${ }^{3}$, Glafiro Torres-Hernández ${ }^{4}$
}

\section{Resumen}

\begin{abstract}
El objetivo de este estudio fue evaluar la influencia del peso al parto de la oveja Pelibuey sobre características de crecimiento predestete de sus corderos en condiciones de clima cálido y húmedo. El estudio fue de tipo retrospectivo y se realizó con información productiva de dos fincas ovinas. Se analizaron 242 observaciones de corderos en la finca 1 y 66 en la finca 2 correspondientes a los pesos al nacer, ganancia de peso predestete y peso de destete ajustado a 61 días. La información se obtuvo de 147 ovejas en la finca 1 y 42 en la finca 2. El modelo estadístico incluyó, en ambas fincas, los efectos fijos de peso de la oveja al parto (por abajo y por encima del promedio), número de parto (primíparas, multíparas), sexo del cordero (hembra y macho) y tipo de nacimiento (simple y múltiple). El peso al parto y el tipo de nacimiento afectaron $(\mathrm{p}<0.05)$ todas las variables dependientes en ambas fincas. El sexo del cordero $(p<0.05)$ influyó sobre el peso de destete en la finca 1. El número de parto afectó el peso al nacer y de destete en la finca $1(\mathrm{p}<0.01)$ y peso al nacer en la finca $2(\mathrm{p}<0.05)$. En conclusión, el peso al parto afectó el comportamiento predestete de los corderos en ambas fincas. Los corderos de ovejas con un peso al parto por encima del promedio fueron más pesados al nacimiento y al destete que los corderos de ovejas con un peso al parto por debajo del promedio. Se confirma que los corderos machos, únicos y provenientes de ovejas multíparas tienen mejor comportamiento predestete con respecto a corderos hembras, múltiples y los de ovejas primíparas, respectivamente.
\end{abstract}

Palabras clave: lactancia; ovinos de pelo; parto; trópico húmedo

\footnotetext{
${ }^{1}$ Universidad Popular de la Chontalpa, Tabasco, México

${ }^{2}$ Campo Experimental Huimanguillo, Instituto Nacional de Investigaciones Forestales, Agrícolas y Pecuarias, Tabasco, México

${ }^{3}$ Universidad Autónoma de Yucatán, Yucatán, México

${ }^{4}$ Colegio de Postgraduados Campus Montecillo, Estado de México, México

${ }^{5}$ E-mail: ponchito34@hotmail.com
} 
The aim of this study was to evaluate the influence of Pelibuey ewe body weight at lambing on pre-weaning performance of their lambs in hot and humid conditions. The study was retrospective and was carried out with productive information on two sheep farms. In total, 242 records of lambs from farm 1 and 66 from farm 2 were analysed for birth weight, pre-weaning body weight gain and weaning weight adjusted to 61 days. The information was obtained from 147 ewes on the farm 1 and 42 on the farm 2 . The statistical model included, in both farms, the fixed effects of ewe body weight at lambing (below and above the average), parity number (primiparous, multiparous), sex of the lamb (female and male) and type of birth (single and multiple). The body weight at lambing and type of lambing affected $(p<0.05)$ all the dependent variables on both farms. The sex of the lamb only affected $(\mathrm{p}<0.05)$ the weaning weight in farm 1 . Parity number influenced birth and weaning weight in farm $1(p<0.01)$ and birth weight in farm $2(p<0.05)$. In conclusion, the ewe body weight at lambing affected the pre-weaning performance of lambs in both farms. Lambs of ewes with a body weight al lambing above average were heavier at birth and at weaning than sheep from ewes with body weight al lambing below average. It is confirmed that male lambs, single lambs and from multiparous sheep they better preweaning performance with respect to female, multiple lambs and those of primiparous sheep, respectively.

Key words: lactation; hair sheep; lambing; wet tropics

\section{INTRODUCCIÓN}

El peso vivo de una oveja es una combinación del tamaño y de la condición corporal, y tiene una relación lineal moderada con la condición corporal (Karakus y Atmaca, 2016; Rodríguez-Valenzuela et al., 2017). El peso vivo del ovino tiene relación con el rendimiento, composición y contenido de energía de la canal (Martínez et al., 1987; Rodríguez-Valenzuela et al., 2017) y el peso al parto en el caso de las hembras reproductoras es importante debido a su influencia sobre la producción de leche y, por lo tanto, en el comportamiento productivo predestete de sus corderos (Sarmiento et al., 1998; Ángeles et al., 2018).

La importancia del peso al nacimiento en los corderos radica en su relación con la salud en el animal adulto (Gardner et al., 2007) y con el peso a la venta (Hinojosa-Cuéllar et al., 2013, 2018); siendo además un componente importante en la productividad de los ovinos de pelo (Magaña-Monforte et al., 2013, 2017; Hinojosa-Cuéllar et al., 2015). En los mamíferos existe un peso al nacimiento óptimo y un rango de confort en el cual se desenvuelve saludablemente el ovino (Gardner et al., 2007). Un bajo peso al nacimiento se asocia con un aumento de mortalidad neonatal, mientras que un alto peso al nacimiento se relaciona con partos distócicos y muerte materna (Dwyer y Bünger, 2012; Lovatt, 2013).

La relación de diversos factores de origen ambiental y genéticos con el peso al nacer del ovino han sido estudiados; entre ellos, el estado nutricional de la oveja al parto, tamaño de la camada, tipo de nacimiento, sexo de la cría, número de parto, y época y año de nacimiento (Gardner et al., 2007; HinojosaCuéllar et al., 2013, 2018). Sin embargo, en las ovejas de pelo, existe limitada información sobre la influencia del peso al parto de la oveja sobre el comportamiento productivo predestete del cordero (Sarmiento et al., 1998; Perón, 2008). El objetivo de este estudio fue evaluar la influencia del peso al parto 
de la oveja Pelibuey sobre características de crecimiento predestete de sus corderos en condiciones de clima cálido y húmedo.

\section{Materiales y Métodos}

\section{Área del Estudio}

La información productiva de los corderos y ovejas fue tomada de los registros de dos fincas comerciales dedicadas a la cría de ovinos Pelibuey localizadas en el estado de Tabasco, México. La finca 1, Quinta Chilla, se encuentra en el km 16.5 de la carretera Villhermosa-Teapa, presenta un clima cálido húmedo con abundantes lluvias en verano, temperatura media anual de $27.0{ }^{\circ} \mathrm{C}$ y precipitación pluvial anual de $2000 \mathrm{~mm}$. La finca 2, El Colibrí, se encuentra ubicada en el km 25 de la carretera Cárdenas-Villahermosa y presenta un clima cálido húmedo con abundantes lluvias en verano, temperatura media anual de $26.7^{\circ} \mathrm{C}$ y precipitación pluvial anual de 1952 mm (Díaz et al., 2006).

\section{Manejo y Alimentación}

Las ovejas se alimentaron con base en pastoreo y un suplemento alimenticio.

En la finca 1, las ovejas gestantes (primeros cuatro meses de la gestación) pastoreaban entre las 08:00 y las 16:00 en praderas con pasto estrella de África (Cynodon plectostachyus), alicia (C. dactylon) y humidicola (Brachiaria humidicola). El mes previo al parto recibieron $600 \mathrm{~g} / \mathrm{animal} / \mathrm{día}$ de un alimento comercial con $14 \%$ de proteína cruda (PC). Las ovejas y sus corderos permanecieron juntos en un corral durante la primera semana posparto, consumiendo alimento comercial (1 kg/oveja/día). A partir de la segunda semana y hasta finalizar la etapa de lactación se mantuvieron en pastoreo entre las 08:00 y las 13:00 y entre las 14:00 y las 16:00, quedando estabuladas en el entretiempo para amamantar a los corderos y recibir $500 \mathrm{~g} /$ oveja/día del alimento comercial (14\%
PC), sal mineral y agua ad libitum. Los corderos se mantuvieron en estabulación durante toda la lactancia, recibiendo un alimento comercial (18\% PC) ad libitum.

En la finca 2, las ovejas gestantes (primeros cuatro meses de la gestación) pastoreaban entre las 08:00 y las 18:00 en praderas con estrella de África (C. plectostachyus) y alicia (C. dactylon). El mes previo al parto recibieron $500 \mathrm{~g} /$ oveja/d de un alimento comercial (15\% de PC). Durante la lactancia pastoreaban entre las 08:00 y las 10:00 y entre las 15:00 y las 18:00. En el entre tiempo estaban en el corral para amamantar a sus corderos y recibir $500 \mathrm{~g} /$ oveja/d del mismo suplemento, sal mineral y agua $a d$ libitum. Los corderos tuvieron acceso a un alimento iniciador con $20 \%$ de PC, agua y sal mineral ad libitum durante toda la lactancia.

Los corderos en ambas fincas fueron pesados al nacimiento (dentro de las primeras $24 \mathrm{~h}$ de vida) y al destete (61 días de edad en promedio). Para ello, se utilizó una balanza de reloj con capacidad de $20 \mathrm{~kg}$ y una sensibilidad de $25 \mathrm{~g}$ (TecnoCor@), México).

\section{Base de Datos}

El estudio fue de tipo retrospectivo observacional. En los corderos se trabajó con la fecha de nacimiento y destete, tipo de nacimiento (simple, doble y triple), sexo (hembra y macho) y año de nacimiento. En la finca 1 se utilizaron 242 registros de pesos de corderos nacidos entre mayo y diciembre de 2008 y en la finca 2 se trabajó con 66 registros de pesos de corderos nacidos entre mayo y octubre de 2014. La información se obtuvo de 147 ovejas en la finca 1 y 42 en la finca 2. En ambas fincas, los registros consultados no incluían la fecha de nacimiento de las ovejas ni el número de parto.

En los corderos, las variables dependientes fueron: peso al nacimiento $(\mathrm{Pn})$, ganancia diaria de peso predestete (Gan), peso al destete $(\mathrm{Pd})$ y peso al destete ajustado a 61 días (Pda). La Gan se calculó como (Pd-Pn)/edad 
Cuadro 1. Análisis de varianza de peso al nacer (Pn), ganancia diaria de peso predestete (Gan) y peso al destete (Pda) en dos rebaños Pelibuey del estado de Tabasco, México

\begin{tabular}{ccccccccc}
\hline \multirow{2}{*}{ Factor } & \multicolumn{9}{c}{ Finca 1 } & \multicolumn{5}{c}{ Finca 2 } \\
\cline { 2 - 9 } M & $\mathrm{gl}$ & $\mathrm{Pn}$ & $\mathrm{Gan}$ & $\mathrm{Pda}$ & $\mathrm{gl}$ & $\mathrm{Pn}$ & $\mathrm{Gan}$ & Pda \\
\hline $\mathrm{TN}$ & 1 & $*$ & $* *$ & $* *$ & 1 & $* *$ & $* *$ & $* *$ \\
$\mathrm{SX}$ & 1 & $\mathrm{~ns}$ & $\mathrm{~ns}$ & $*$ & 1 & $\mathrm{~ns}$ & $\mathrm{~ns}$ & $\mathrm{~ns}$ \\
$\mathrm{NP}$ & 1 & $* *$ & $\mathrm{~ns}$ & $* *$ & 1 & $*$ & $\mathrm{~ns}$ & $\mathrm{~ns}$ \\
$\mathrm{M}$ TN & 1 & $\mathrm{~ns}$ & $\mathrm{~ns}$ & $\mathrm{~ns}$ & 1 & $\mathrm{~ns}$ & $\mathrm{~ns}$ & $\mathrm{~ns}$ \\
$\mathrm{M}$ SX & 1 & $\mathrm{~ns}$ & $\mathrm{~ns}$ & $\mathrm{~ns}$ & 1 & $\mathrm{~ns}$ & $\mathrm{~ns}$ & $\mathrm{~ns}$ \\
$\mathrm{M}$ NP & 1 & $\mathrm{~ns}$ & $\mathrm{~ns}$ & $\mathrm{~ns}$ & 1 & $\mathrm{~ns}$ & $\mathrm{~ns}$ & $\mathrm{~ns}$ \\
Error & 234 & & & & 58 & & & \\
Total & 241 & & & & 65 & & & \\
$\mathrm{R}^{2}$ & & 0.35 & 0.24 & 0.33 & & 0.37 & 0.32 & 0.35 \\
\hline
\end{tabular}

$\mathrm{M}=$ grupo de peso al parto de la oveja; $\mathrm{TN}=$ tipo de nacimiento; $\mathrm{SX}=$ sexo del cordero; $\mathrm{NP}=$ número de parto; $M * N P, M^{*} T N$ y $M^{*} S X$ interacciones de primer orden; $g l=$ grados de libertad; $R^{2}=$ coeficiente de determinación; ** Significativo $(p<0.01)$, * Significativo $(p<0.05), n s=$ no significativo

al destete en días. El peso al destete se ajustó a 61 días con la fórmula: $\mathrm{Pda}=(\mathrm{Gan} \times \mathrm{x} 6)$ $+\mathrm{Pn}$, donde $\mathrm{Pda}=$ peso al destete ajustado a 61 días de edad $(\mathrm{kg}), \mathrm{Gan}=$ ganancia diaria de peso predestete $(\mathrm{kg})$ y $61=$ edad al destete (días) como constante. Los corderos con tipo de nacimiento doble o triple se agruparon en una sola clase (múltiples) debido al bajo número de observaciones de corderos con tipo de nacimiento triple.

La información productiva de las ovejas en las dos fincas correspondió a años distintos, de allí que se realizaron análisis estadísticos independientes. El modelo que describió la varianza de las variables Pn, Gan y Pda fue el mismo para ambas fincas. Las variables incluidas como independientes para cada finca fueron: ovejas con un peso al parto por abajo o por encima del peso promedio, tipo de nacimiento (simple y múltiple), sexo del cordero (hembra y macho) y número de parto (primíparas y multíparas). Además, todas las interacciones posibles de primer orden asociadas con la categoría de peso (por arriba o por abajo). Todos los análisis se realizaron con el procedimiento GLM (SAS, 2002).

\section{Resultados}

Los resultados del análisis de varianza se presentan en el Cuadro 1 y las medias de cuadrados mínimos por cada variable independiente en cada finca en los cuadros 2 y 3 . Las medias generales $( \pm \mathrm{DE})$ estimadas de Pn, Gan y Pda fueron $2.9 \pm 0.69,0.165 \pm$ 0.055 y $13.0 \pm 3.6 \mathrm{~kg}$, respectivamente, en la finca $1, y$ de $2.6 \pm 0.65,0.165 \pm 0.050$ y 12.3 $\pm 3.49 \mathrm{~kg}$ en la finca 2 , respectivamente. Los promedios en los pesos al parto $( \pm \mathrm{DE})$ fueron de $41.2 \pm 5.1$ y $34.2 \pm 7.1 \mathrm{~kg}$ en las fincas 1 y 2 , respectivamente. 
Cuadro 2. Medias de cuadrados mínimos ( \pm error estándar) de peso al nacer (Pn), ganancia diaria de peso predestete (Gan) y peso al destete ajustado (Pda) en un rebaño (Finca 1) Pelibuey del estado de Tabasco, México

\begin{tabular}{lcccc}
\hline Factor & $\mathrm{N}$ & $\mathrm{Pn}$ & Gan & Pda \\
\hline $\mathrm{M} \quad$ Abajo & 147 & $2.8 \pm 0.03$ & $0.150 \pm 0.004$ & $12.0 \pm 0.2$ \\
\multicolumn{1}{c}{ Arriba } & 95 & $3.0 \pm 0.04$ & $0.180 \pm 0.005$ & $14.0 \pm 0.3$ \\
$\mathrm{TN}$ & & $* *$ & $* *$ & $* *$ \\
Simple & 63 & $3.1 \pm 0.05$ & $0.186 \pm 0.006$ & $14.4 \pm 0.4$ \\
$\quad$ Múltiple & 179 & $2.8 \pm 0.03$ & $0.144 \pm 0.003$ & $11.6 \pm 0.2$ \\
$\mathrm{SX}$ & & $\mathrm{ns}$ & $\mathrm{ns}$ & $*$ \\
Hembra & 129 & $2.9 \pm 0.03$ & $0.159 \pm 0.004$ & $12.6 \pm 0.3$ \\
$\quad$ Macho & 113 & $3.0 \pm 0.03$ & $0.172 \pm 0.004$ & $13.4 \pm 0.3$ \\
$\mathrm{NP}$ & & $* *$ & $\mathrm{~ns}$ & $* *$ \\
Primíparas & 133 & $2.4 \pm 0.04$ & $0.161 \pm 0.005$ & $12.6 \pm 0.3$ \\
Multíparas & 109 & $3.4 \pm 0.03$ & $0.169 \pm 0.004$ & $13.7 \pm 0.3$ \\
\hline
\end{tabular}

$\mathrm{M}=$ peso de la oveja al parto; $\mathrm{TN}=$ tipo de nacimiento; $\mathrm{SX}=$ sexo del cordero; $\mathrm{NP}=$ número de parto; $\mathrm{N}=$ número de observaciones; ** Significativo $(p<0.01)$, * Significativo $(p<0.05), n s=$ no significativo Valores en kilogramos

Cuadro 3. Medias de cuadrados mínimos ( \pm error estándar) de peso al nacer (Pn), ganancia diaria de peso predestete (Gan) y peso al destete ajustado (Pda) en un rebaño (Finca 2) Pelibuey del estado de Tabasco, México

\begin{tabular}{|c|c|c|c|c|}
\hline Factor & $\mathrm{N}$ & $\mathrm{Pn}$ & Gan & Pda \\
\hline M & & $* *$ & $* *$ & $* *$ \\
\hline Abajo & 38 & $2.1 \pm 0.14$ & $0.130 \pm 0.012$ & $10.0 \pm 0.8$ \\
\hline Arriba & 28 & $3.0 \pm 0.17$ & $0.192 \pm 0.014$ & $14.7 \pm 0.9$ \\
\hline $\mathrm{TN}$ & & $* *$ & $* *$ & $* *$ \\
\hline Simple & 24 & $2.8 \pm 0.16$ & $0.188 \pm 0.013$ & $14.3 \pm 0.9$ \\
\hline Múltiple & 42 & $2.2 \pm 0.12$ & $0.135 \pm 0.010$ & $10.4 \pm 0.7$ \\
\hline SX & & ns & ns & ns \\
\hline Hembra & 31 & $2.5 \pm 0.14$ & $0.155 \pm 0.012$ & $12.0 \pm 0.8$ \\
\hline Macho & 35 & $2.5 \pm 0.11$ & $0.167 \pm 0.009$ & $12.7 \pm 0.6$ \\
\hline NP & & $*$ & $\mathrm{~ns}$ & $\mathrm{~ns}$ \\
\hline Primíparas & 8 & $2.3 \pm 0.20$ & $0.165 \pm 0.016$ & $12.4 \pm 1.1$ \\
\hline Multíparas & 58 & $2.7 \pm 0.09$ & $0.157 \pm 0.008$ & $12.3 \pm 0.5$ \\
\hline
\end{tabular}

$\mathrm{M}=$ peso de la oveja al parto (abajo o arriba del promedio); $\mathrm{TN}=$ tipo de nacimiento; $\mathrm{SX}=$ sexo del cordero; $\mathrm{NP}=$ número de parto; $\mathrm{N}=$ número de observaciones; ${ }^{*}$ Significativo $(\mathrm{p}<0.01),{ }^{*}$ Significativo $(\mathrm{p}<0.05), \mathrm{ns}=$ no Valores en kilogramos 
El peso de la oveja al parto afectó las tres variables dependientes estudiadas en ambas fincas (Cuadro 1). En los cuadros 2 y 3 se puede observar que los corderos de ovejas con peso al parto por abajo del promedio de peso al parto tuvieron menor Pn, Gan y Pda con relación a los corderos provenientes de ovejas con peso al parto por encima del promedio del peso al parto. Así mismo, los corderos de origen múltiple presentaron menores valores en todas las variables comparados con corderos únicos $(\mathrm{p}<0.01)$. El sexo de los corderos solamente afectó el Pda en la finca 1. El número de parto afectó el Pn en ambas fincas y el Pda en la finca 1 . Los machos tuvieron mayor Pda que las hembras y los corderos provenientes de ovejas multíparas superior Pn y Pda en la finca 1 y mayor Pn en la finca 2 comparados con los corderos de primíparas.

\section{Discusión}

Las medias de Pn están dentro de los límites reportados ( 2.0 y $3.5 \mathrm{~kg})$ en la raza Pelibuey (Herrera et al., 2008; OlivaHernández y García-Osorio, 2016; AguilarMartínez et al., 2017). Las Gan de los corderos provenientes de ambas fincas fueron mayores a las obtenidas en corderos Pelibuey destetados a 60 días (Hinojosa-Cuéllar et al., 2012; Oliva-Hernández y García-Osorio 2016) y 90 días de edad (Macías-Cruz et al., 2012). Estas diferencias en valores pudieran ser atribuidas a las diferencias en el manejo nutricional de las ovejas y corderos.

\section{Peso al Parto}

El peso de la oveja al parto afectó el Pn, Gan y Pda de los corderos en ambas fincas. El mayor Pn de los corderos provenientes de ovejas con un mayor peso al parto sugiere que este tipo de ovejas probablemente tuvieron mayores reservas corporales para apoyar el crecimiento fetal con respecto a los corderos de ovejas con un menor peso al parto. En la región tropical, disponer de ma- yores reservas corporales durante la última fase de la gestación es determinante, sobre todo en sistemas de alimentación que se apoyan en el pastoreo, ya que en este sistema de alimentación las ovejas se exponen a la influencia negativa de una alta temperatura ambiente la cual puede disminuir su eficiencia alimenticia y con ello reducir las posibilidades de cubrir sus necesidades de nutrientes (Marai et al., 2008; Salah et al., 2014).

Es importante señalar la influencia que ejerció el peso al parto sobre Gan y Pda. Corderos cuyas madres tuvieron un peso al parto por arriba del promedio de peso al parto del grupo mostraron una Gan y Pda superior en 20.0 y $16.7 \%$ en la finca 1 y 47.7 y $47.0 \%$ en la finca 2 , al de corderos cuyas madres tuvieron un peso al parto por abajo del promedio de peso al parto (cuadros $2 \mathrm{y}$ 3). El peso al parto de la oveja tiene una relación positiva con la producción total de leche (Ángeles et al., 2018), lo que pudiera explicar la mayor Gan y Pda de corderos de madres con mayor peso al parto. Segura-Correa et al. (1996), al estudiar la productividad de ovejas Pelibuey y Blackbelly bajo condiciones extensivas de manejo en México, encontraron una menor productividad y prolificidad en ovejas con menor peso al promedio de peso del grupo.

\section{Tipo de Nacimiento}

En los mamíferos placentarios el espacio uterino tiene una capacidad finita para gestar. A medida que aumenta el número de corderos en la camada el peso al nacimiento individual disminuye (Gardner et al., 2007). En el presente estudio el tipo de nacimiento afectó el Pn, la Gan y el Pda en ambas fincas (Cuadro 1). Las diferencias a favor de corderos de origen simple respecto a los múltiples fueron de $0.3,0.042$ y $2.8 \mathrm{~kg}$ para $\mathrm{Pn}$, Gan y Pda, respectivamente, en la finca $1 \mathrm{y}$ de $0.6,0.053$ y $3.9 \mathrm{~kg}$ para las mismas variables, respectivamente en la finca 2 (cuadros 2 y 3 ). Diversos estudios concuerdan con la superioridad del comportamiento predestete de corderos únicos en relación con los múlti- 
ples (Benyi et al., 2006; Gardner et al., 2007; Hinojosa-Cuéllar et al., 2013, 2018).

El mayor Pn en crías únicas se debe, en parte, a que el crecimiento intrauterino del feto único no tiene restricción de espacio ni competencia por nutrientes (McCoard et al., 2017). Además, la menor Gan y Pda de los corderos de tipo múltiple está en estrecha relación con el menor peso del músculo esquelético que presentan cuando se encuentran como fetos próximos al nacimiento, un menor consumo de leche y con la habilidad de la oveja para amamantar a más de una cría (Cardellino y Benson, 2002; Sales et al., 2013; OlivaHernández y García-Osorio, 2016).

\section{Sexo del Cordero}

El sexo del cordero afectó el Pda en la finca 1 (Cuadro 2). El peso al destete usualmente es mayor en corderos machos que en hembras, y esto se observa también en corderos Pelibuey (Carrillo et al., 1987; Carrillo y Segura, 1993). En corderos de razas de pelo híbridas se han encontrado diferencias de $10.4 \%$ a favor de los machos (HinojosaCuéllar et al., 2011). Este mayor crecimiento se asocia con el incremento en la concentración de testosterona plasmática que se libera por las gónadas después del nacimiento (O’Shaughnessy, 2015; Montiel-Olguín et al., 2016).

\section{Número de Parto}

El número de parto influyó $(\mathrm{p}<0.01)$ en el Pn y Pda en la finca 1 y $\operatorname{Pn}(\mathrm{p}<0.05)$ en la finca 2 (Cuadro 1). Los corderos de hembras multíparas mostraron superioridad sobre corderos de hembras primíparas en estas características (Cuadros 2 y 3). Estos resultados coinciden con otros trabajos en la raza Blackbelly y Pelibuey, respectivamente (González-Domínguez et al., 2016; HinojosaCuéllar et al., 2018). La menor productividad de las ovejas primíparas pudiera ser atribuida a que este tipo de hembras tienen menor edad, menor peso vivo y no han alcanzado la madurez, por lo que siguen creciendo durante la gestación y genera una competencia por los nutrientes disponibles entre el cuerpo materno y su útero grávido (Bermejo et al., 2010; García-Osorio et al., 2017). Por otro lado, las ovejas con dos años producen más leche que las ovejas de un año (Cardellino y Benson, 2002), lo cual contribuye a explicar estas diferencias.

\section{Conclusión}

- Los resultados señalan la importancia de poner énfasis en el manejo nutricional de la oveja Pelibuey en pastoreo para aumentar el peso al parto y mejorar el comportamiento predestete del cordero.

- Los corderos de ovejas con un peso al parto por encima del promedio fueron más pesados al nacimiento y al destete que los corderos de ovejas con un peso al parto por debajo del promedio.

- Se confirma que los corderos machos, únicos y provenientes de ovejas multíparas tienen mejor comportamiento predestete con respecto a corderos hembras, múltiples y los de ovejas primíparas, respectivamente.

\section{Agradecimientos}

Los autores agradecen al Arq. Juan Carlos Domínguez García y al Ing. Walter Lanz propietarios de las fincas «Quinta Chi1la» $\mathrm{y}$ «El Colibrí», respectivamente, por haber permitido tomar la información de los registros productivos de sus rebaños para elaborar el presente estudio.

\section{Literatura CitAda}

1. Aguilar-Martínez $C U$, BerruecosVillalobos JM, Espinoza-Gutiérrez B, Segura-Correa JC, Valencia-Méndez J, Roldán-Roldán A. 2017. Origen, historia y situación actual de la oveja Pelibuey en México. Trop Subtrop Agroecosyst 20: 429-239. 
2. Ángeles HJC, Radic SS, Vera AMA, Echeverría PRA, Castelán-Ortega OA, Ramírez PAH, González RM. 2018. Effect of live weight pre-and post-lambing on milk production of East Friesian sheep. Ital J Anim Sci 17: 184-194. doi: 10.1080/ 1828051X.2017.-1349536

3. Benyi K, Norris D, Karbo NK, Gomo K $\boldsymbol{A}$. 2006. Effects of genetic and environmental factors on pre-weaningpost-weaning growth in West African crossbred sheep. Trop Anim Health Prod 38: 547-554. doi: 10.1007/s11250-0064416-2

4. Bermejo LA, Mellado M, Camacho A, Mata J, Arévalo JR, de Nascimento L. 2010. Factors influencing birth and weaning weight in Canarian hair lambs. J Anim Vet Adv 37: 273-275. doi: 10.1080/09712119.2010.9707140

5. Cardellino RA, Benson ME. 2002. Lactation curves of commercial ewes rearing lambs. J Anim Sci 80: 23-27. doi: $10.2527 / 2002.80123 \mathrm{x}$

6. Carrillo AL, Velázquez MA, Ornelas GT. 1987. Algunos factores ambientales que afectan el peso al nacer y al destete de corderos Pelibuey. Tec Pecu Mex 25:289-295.

7. Carrillo AL, Segura-Correa JC. 1993. Environmental and genetic effects on pre-weaning growth performance of hair sheep in México. Trop Anim Health Prod 25: 173-178. doi: 10.1007/BF02236237

8. Díaz PG, Ruíz CJA, Medina GG, Cano GMA, Serrano AV. 2006. Estadísticas climáticas básicas del estado de Tabasco (periodo 1961-2003). Libro Técnico 12. Veracruz, México: Instituto Nacional de Investigaciones Forestales, Agrícolas y Pecuarias.

9. Dwyer CM, Bünger L. 2012. Factors affecting dystocia and offspring vigour in different sheep genotypes. Prev Vet Med 103: 257-64. doi: 10.1016/ j.prevetmed.2011.09.002

10. García-Osorio I del C, OlivaHernández J, Osorio-Arce MM, Torres-Hernández G, Hinojosa-Cuéllar
JA, González-Garduño R. 2017. Influencia materna en el crecimiento predestete y características de la canal de corderos de pelo. Ecosist Recur Agropec 4: 51-63. doi: 10.19136/ era.a4n10.818

11. Gardner DS, Buttery PJ, Daniel Z, Symonds ME. 2007. Factors affecting birth weight in sheep: maternal environment. Reproduction 133: 297 307. doi: 10.1530/REP-06-0042

12. González-Domínguez G, HinojosaCuéllar JA, Oliva-Hernández J, Torres-Hernández G, Segura-Correa JC, González-Garduño R, GarcíaOsorio I. 2016. Análisis del comportamiento predestete de corderos Barbado Barriga Negra en clima cálido húmedo. Nova Scientia 8: 181-197. doi: 10.21640/ ns.v8i17.603

13. Herrera J, Pulgarón P, Noda AC. 2008. Comportamiento productivo de ovinos Pelibuey en un sistema con bajos insumos. Cuban J Agr Sci 42: 45-49.

14. Hinojosa-Cuéllar JA, TorresHernández G, Oliva-Hernández J, Aranda-Ibáñez E, Segura-Correa JC, González-Camacho JM. 2011. Preweaning performance of lambs from purebred and crossbred hair ewes under humid tropical conditions of Tabasco, Mexico. J Anim Vet Adv 10: 3149-3154. doi: 10.3923/javaa.2011.3149.3154.

15. Hinojosa-Cuéllar JA, OlivaHernández J, Torres-Hernández G, Segura-Correa JC, Aranda-Ibañez, E, González-Camacho JM. 2012. Factores que afectan el crecimiento predestete de corderos Pelibuey en el trópico húmedo de México. Univ Cienc 28: 163-171. doi: 10.19136/era.a28n2.24

16. Hinojosa-Cuéllar JA, Oliva-Hernández J, Torres-Hernández G, SeguraCorrea JC. 2013. Comportamiento productivo de corderos $\mathrm{F}_{1}$ Pelibuey x Blackbelly y cruces con Dorper y Katahdin en un sistema de producción del trópico húmedo de Tabasco, México. Arch Med Vet 45: 135-143. 
17. Hinojosa-Cuéllar JA, Oliva-Hernández J, Torres-Hernández, G, SeguraCorrea JC, González-Garduño R. 2015. Productividad de ovejas $F_{1}$ Pelibuey x Blackbelly y sus cruces con Dorper y Katahdin en un sistema de producción del trópico húmedo de Tabasco, México. Arch Med Vet 47: 167-174.

18. Hinojosa-Cuéllar JA, OlivaHernández J, Torres-Hernández G, Segura-Correa JC. 2018. Crecimiento pre y posdestete de corderos Pelibuey en clima cálido húmedo. Nova Scientia 10: 328-351. doi: 10.21640/ns.v10i20.-1335

19. Karakus F, Atmaca M. 2016. The effect of ewe body condition at lambing on growth of lambs and colostral specific gravity. Arch Anim Breed 59: 107-112. doi: 10.5194/aab-59-107-2016

20. Lovatt F. 2013. Ovine obstetrics: aiming for a healthy ewe and lamb. Vet Rec 172: 552-553. doi: 10.1136/vr.f3185

21. Macías-Cruz U, Alvarez-Valenzuela FD, Olguín-Arredondo HA, MolinaRamírez L, Avendaño-Reyes L. 2012. Ovejas Pelibuey sincronizadas con progestágenos y apareadas con machos de razas Dorper y Katahdin bajo condiciones estabuladas: producción de la oveja y crecimiento de los corderos durante el período predestete. Arch Med Vet 44: 29-37. doi: 10.4067/S0301-732X2012000100005

22. Magaña-Monforte JG, Huchin-Cab M, Ake-López RJ, Segura-Correa JC. 2013. A field study of reproductive performance and productivity of Pelibuey ewes in Southeastern Mexico. Trop Anim Health Prod 25: 173-178. doi: 10.1007/ s11250-013-0431-2

23. Magaña-Monforte JG, Segura-Correa JC, Aké-López JR, Chay-Canul AJ. 2017. Efecto del peso de la borrega al parto sobre la eficiencia al destete de ovejas de pelo en Yucatán, México. En: Memorias XI Seminario Internacional de Producción de Ovinos en el Trópico. Tabasco, México.
24. Marai IFM, El-Darawany AA, Fadiel A, Abdel-Hafez AM. 2008. Reproductive performance traits as affected by heat stress and its alleviation in sheep. Trop Subtrop Agroecosyst 12: 77-84.

25. Martínez AA, Bores QR Castellanos $\boldsymbol{R} A \boldsymbol{F}$. 1987. Zoometría y predicción de la composición corporal de la borrega Pelibuey. Tec Pecu Mex 25: 72-84.

26. McCoard S, Sales F, Sciascia Q. 2017. Invited review: impact of specific nutrient interventions during mid-to-late gestation on physiological traits important for survival of multiple-born lambs. Animal 11: 1727-1736. doi: 10.1017/S1751731117000313

27. Montiel-Olguín LJ, Vera-Ávila HR, Villagómez-Amezcua E, CastañedaRodríguez V, Cárdenas-León M, Jiménez-Severiano H. 2016. Desarrollo del eje reproductivo endocrino en corderos de pelo. Rev Mex Cienc Pecu 7: 341-360. doi: 10.22319/rmcp.v7i3.4214

28. Oliva-Hernández J. García-Osorio I. 2016. A note on the changes in the preweaning growth of Pelibuey lambs. Arch Med Vet 48: 293-298.

29. O'Shaughnessy P. 2015. Testicular development. In: Plant TM, Zeleznik AJ (eds). Knobil and Neill's physiology of reproduction. $4^{\text {th }}$ ed. USA: Elsevier. $p$ 567-594.

30. Perón N. 2008. Crecimiento del ovino Pelibuey en Cuba. Revisión bibliográfica. Cienc Tecnol Ganadera 2: 117-131.

31. Rodríguez-Valenzuela E, Chay-Canul AJ, García-Herrera RA, SalazarCuytun R, Piñeiro-Vázquez AT, Casanova-Lugo F, et al. 2017. Prediction of carcass energy content of Pelibuey ewes by ultrasound measurements. Ecosist Recur Agropec 4: 543-548. doi: 10.19136/era.a4n12.1226

32. Salah N, Sauvant D, Archimède $H$. 2014. Nutritional requirements of sheep, goats and cattle in warm climates: a meta-analysis. Animal 8: 1439-1447. doi: $10.1017 / \mathrm{S} 1751731114001153$ 
33. Sales F, Pacheco D, Blair H, Kenyon P. McCoard S. 2013. Muscle free amino acid profiles are related to diffe-rences in skeletal muscle growth between single and twin ovine fetuses near term. Springerplus 2: 483. doi: 10.1186/2193-1801-2-483

34. Sarmiento FL, Segura CJC, Rojas RO. 1998. Cambios de peso en ovejas Pelibuey y Blackbelly y su relación con el peso vivo predestete de las crías. Agrociencia 32: 119-123
35. [SAS] Statistical Analysis System. 2002. SAS/STAT User's guide (Release 9.0) Cary, North Carolina, USA: SAS Institute Inc.

36. Segura-Correa JC, Sarmiento L. Rojas O. 1996. Productivity of Pelibuey and Blackbelly ewes in Mexico under extensive management. Small Ruminant Res 21: 57-62. doi: 10.1016/09214488(95)00814-4 